\title{
HIV Sero Status and Associated Factors Among HIV-Exposed Infants' in Selected Health Facilities in Sidama Zone, Southern Ethiopia
}

\author{
Yirgalem Yosef ${ }^{1, ~ *}$, Bosena Tebeje ${ }^{2}$, Jophine Joseph $^{2}$, Seblework Abeje ${ }^{3}$ \\ ${ }^{1}$ Department of Midwifery, College of Health Science, Wolkite University, Wolkite, Ethiopia \\ ${ }^{2}$ School of Nursing and Midwifery, Faculty of Health Science, Institute of Health, Jimma University, Jimma, Ethiopia \\ ${ }^{3}$ Department of Biochemistry, College of Health Science, Wolkite University, Wolkite, Ethiopia \\ Email address: \\ Yirgalem.yosef@wku.edu.et (Y. Yosef), bosenatebeje@yahoo.co.uk (B. Tebeje), jophinkjoseph@yahoo.co.in (J. Joseph), \\ Seblework.abeje@wku.edu.et (S. Abeje) \\ ${ }^{*}$ Corresponding author
}

\section{To cite this article:}

Yirgalem Yosef, Bosena Tebeje, Jophine Joseph, Seblework Abeje. HIV Sero Status and Associated Factors Among HIV-Exposed Infants' in Selected Health Facilities in Sidama Zone, Southern Ethiopia. Journal of Family Medicine and Health Care. Vol. 6, No. 3, 2020 , pp. $70-77$. doi: $10.11648 /$ j.jfmhc.20200603.13

Received: January 15, 2020; Accepted: May 27, 2020; Published: June 9, 2020

\begin{abstract}
MTC transmission of HIV is a major public health challenge in Ethiopia; however there were a shortage of evidence on HIV sero status of exposed infants in Sidama zone, Southern Ethiopia. Therefore, this study aimed to assess, the HIV sero status and associated factors among HIV-exposed infants in selected public health facilities. A cross-sectional study was conducted in 14 public health facilities at Sidama zone SNNPR state, Ethiopia from May 1- 30/2019Gc. Medical record cards of HIV-exposed infants and their mothers enrolled from January 2014 to January 2018 was extracted using data extraction checklist. Magnitude and associated factors of HIV sero status of exposed infants was computed using SPSS version 21.0 software. A total of 203 HIV-exposed infants who had HIV DNA/PCR test results were included in the study. The overall prevalence of HIV among HIV exposed infants were nearly $9 \%$ (95\% CI: 4.4, 12.8) Infants born from mothers' with baseline CD4+ counts of mother less than 350, $(\mathrm{AOR}=5.629 ; 95 \%$ CI: $1.454,21.79)$, Mothers who had WHO clinical stage I and II, (AOR $=4.975 ; 95 \%$ CI: $1.342,18.446$ ), and Poor ART adherence (AOR=4.302; 95\% CI: 1.100, 16.823) had an increased odds of HIV infection comparing to their counterparts. Conclusion and recommendation: The prevalence of HIV infection among infants born to HIV infected mothers was high in the study area. Baseline CD4+ counts, WHO clinical staging, and ART adherences of mothers are associated with their infants HIV status. Therefore, stakeholders including health care workers working at PMTCT centers should make create awareness on the effects of poor ART adherence and undertaking further rigorous longitudinal studies are recommended.
\end{abstract}

Keywords: HIV Sero Status, HIV Exposed Infants, DNA/PCR Test, OPTION B+ Strategy, Ethiopia

\section{Background}

MTCT of HIV is transmission of human immunodeficiency virus from HIV infected mother to exposed infants during pregnancy, childbirth and post-natal period of the pregnancy $[1,2]$. HIV infected infants' results in lifelong chronic health problems, such as premature morbidity and morbidity of the infants [4]. According to the United Nations Programmer on HIV/AIDS reported that in 2016 an estimated 160,000 children were newly infected, and an projected 3.1 million children were living with HIV worldwide and there were nearby to 500,000 children infected with HIV through infected mother to exposed infants transmission each year [4]. And also According to USAID 2017 report, there were just about 1.4 million pregnant women, and 1.8 million children were livelihood with HIV/AIDS. It is also projected that 1.8 million individuals turn out to be newly infected, of which 180,000 will be under 
15 years old children [10].

The Plan in the direction of eradicating new HIV infections among children by 2015 and provide an ultimate care to their mothers launched in 2011 , and set a sequences of targets for 2015 to reduce new HIV infections among children through $90 \%$ in 2015 . The successful international pressure groups recommended that all pregnant and breastfeeding women to be on antiretroviral therapy, nevertheless of CD4 T-cell count or clinical staging established the phase for the World Health Organization's properly named "Treat All" guidelines, eradicated numerous longstanding obstacles to treat HIV/AIDS [11].

In Ethiopia there were nearly 90,000 HIV positive pregnant women who live with HIV AIDS and a projected 14,000 HIV positive births in 2017 [12]. In similar years the pooled prevalence of mother to child transmission of HIV was $9.93 \%$ (3). To eradicate new HIV Infections among children, the Ethiopian MOH adopted PMTCT shift. In “August 2012, the country adopted WHO Prevention of mother to child transmission programmatic shift, option $\mathrm{B}+$ strategy. The program recommends lifelong antiretroviral therapy (ART) for all HIV-positive women who are pregnant and breastfeeding, regardless of the CD4 count or clinical staging [13]. The post-2015 HIV priorities were high impact interventions that radically reduce the annual new infection and save many lives [14]. Studies conducted in Ethiopia have also shown that the magnitude of HIVpositive children in Ethiopia is still high [8]. Moreover, reducing infant death due to infected mother to exposed infants' transmission of HIV has been the matter of concern for Ethiopian Ministry of health for years. Ethiopia have adopted PMTCT programmatic shift, option B+ strategy from World Health Organization (WHO) as a comprehensive approach to reduce the transmission of HIV/AIDS to infants during pregnancy labour and delivery and through breast feeding [13]. But, detailed information on rate of Mother to child transmission of HIV/AIDS after end of follow up of exposed infant remains in short in Ethiopia, in spite of its harmful effects on child health [15]. Especially, the HIV sero status and the factors were not well studied in Ethiopia particularly in Sidama zone. Therefore, this study was conducted to determine the HIV sero status of infants and associated factors among HIVexposed infants in selected health facilities in Sidama zone south.

The study would provide helpful information on HIV sero status and associated factors among exposed infant in Sidama zone. In addition; it may contribute to reduce infant and maternal morbidity and mortality associated with HIV/AIDS. It may also provide helpful information to modify and design nursing intervention for HIV exposed infants to eliminating new HIV infection among children while keeping children living with HIV alive and well. Additionally, it may give a base line data for interested researchers, policy makers and scientific knowledge.

\section{Methods and Materials}

\subsection{Study Area and Period}

The study was done in public health facilities found in Sidama zone, SNNPR, in the southern part of Ethiopia from May 1 to 30/2019Gc. Sidama Zone, is located in the eastern part of SNNPR covers about $6,538 \mathrm{~km}^{2}$. It is one of 15 zones found in SNNPR, Ethiopia. In 2017 Total population of Sidama zone around 4.5 million and there are around 0.75 million house hold. This zone is located between $3^{\circ} 14^{\prime} \mathrm{N}$ latitude and $33^{\circ} 48^{\prime} \mathrm{E}$ and it has 33 districts and two selfadministration towns. This zone is bordered on the south by the Oromia Region, except for a short stretch in the middle where it shares a border with Gedeo zone, on the west by the Bilate River, which separates it from Wolieta zone, and on the north and east by the Oromia Region [24]. Hawassa is the capital city of Sidama and SNNPR, and situated $279 \mathrm{~km}$ from Addis Ababa capital city of Ethiopia. Sidama zone is the largest most populous and urbanizing zone in the SNNPR state of Ethiopia. Among adults ages 15-64 years HIV prevalence in urban area of SNNPR was $1.8 \%$ [2]. Sidama zone is one of the HIV/AIDS-affected regions in SNNPR state. In this zone there were total 361 HIV exposed infant and from total exposed 203 infants had DNA/ PCR test results from January 2014 to January 2018Gc. This zone has twelve primary hospitals, one zonal and one general public hospital [30].

\subsection{Study Design, Sampling Technique and Procedures}

Institution based cross sectional study design was used to investigate HIV sero status and associated factors among HIV-exposed infants using a census sampling technique. Participants for this study were HIV-exposed infants medical registration card which had DNA / PCR tested result enrolled from January 2014 to January 2018Gc. Study was conducted in selected health facilities which had been providing PMTCT service. From the total health facilities found in Sidama zone, fourteen [14] health facilities had ART clinic and had been providing PMTCT service linked with other health facilities to address services for the whole catchment area. In this zone from 2014 to 2018 there were 203 HIV positive women and exposed infants pair (mother -infant pair) who had PCR test result enrolled in PMTCT of selected health facilities. The data were extracted retrospective from PMTCT log book and maternal medical registration card by using data extraction checklist.

\subsection{Study Population and Source Population}

All HIV-exposed infants' medical records card having DNA/ PCR test result and enrolled in selected health facilities from January 2014 to January $2018 \mathrm{Gc}$ used as a study population. Medical records of HIV-exposed infants and their mothers enrolled from January 2014 to January 2018 in the study institutions were considered as a source population.

Eligibility criteria- All HIV-exposed infants medical 
registration card which had HIV PCR test result were included while Exposed infants transfer out, lost and stopped treatment were excluded from the study.

Study Variables: Dependent variable- HIV sero-status of Baby (positive/negative) and the independent variables were Socio-demographic factors, Clinical characteristics of the mother, Obstetrics characteristics of the mother, and clinical Characteristics of exposed infants.

Operational definitions-Exposed infants HIV status: If the DNA/PCR test result indicated positive or negative for HIV during the follow-up period of 18 months as indicated by the infant registration card (15). While, HIV Positive: when the DNA/PCR test result for exposed infants indicated positive for HIV during the follow-up period of 18 months and HIV Negative: when the DNA/PCR test result for exposed infants indicated negative for HIV during the follow-up period of 18 months.

\subsection{Data Collection Instruments and Procedures}

The data were extracted from PMTCT registration log book through using; data extraction format adopted from the national standard HIV exposed infant follow up formats [24]. All HIV-exposed infants and their mothers who started the Prevention of mother to child transmission service in selected health facilities during the study period were included in the study. HIV exposed infants and their mothers with incomplete data because of transfer out, lost and stopped treatment were excluded from the study. Some important variables incomplete in registration logbook during filtration of data were counted as missing variable. Data was collected by trained and experienced clinical nurses and diploma midwifes who had at least 2 years of work experience in ART clinic.

\subsection{Data Quality Management}

Data quality assurance mechanisms was carefully developed and implemented at various stages of the study. The quality of data may be affected at different points unless proper measures were taken throughout the study time. For all study areas, three data collectors and two supervisors who had ability to communicate in Amharic and local language were recruited after one day training about data collection technique. To ensure data quality, preliminary assessment was done to include all variables registered in infants PMTCT follow up log books and maternal medical registration card in similar health facility. At the end of the day filled questionnaires were checked for completeness and consistency of information by the supervisor and errors were manually edited. Any ambiguity and other problems of data collectors were addressed. Cronbach's alpha and collinearity was checked.

\subsection{Data Processing and Analysis Procedures}

Data cleaning was performed to check for accuracy, consistencies of the data. The data collection was undertaken under rigorous daily checking to identify and correct errors. The investigator entered the data using Epi Data version 3.1 and exported to SPSS 21 statistical package for analysis. Descriptive statistics (frequency and percentage) were used to describe sero status of exposed infants and associated factors. Then bivariate logistic regression analysis was done to see the crude association between the independent variables and the dependent variables and the strength of association were expressed the odds ratio (OR). Eventually, results from bivariate analysis of $\mathrm{p}<0.25$ were moved to multivariate analysis and done through backward variable selection logistic regression methods to control the effects of confounding and to identify predictors of HIV sero status of exposed infants. A P value of $<0.05$ used to determine the significance of association.

\section{Results}

Magnitude of HIV among exposed infants: The finding of this study show that among infants who had DNA/PCR test results, HIV sero status positive infants were 18 (8.9\%) CI: (4.4, 12.8) and HIV sero status negative were 185 (91.1\%) during the study period (Figure 1).

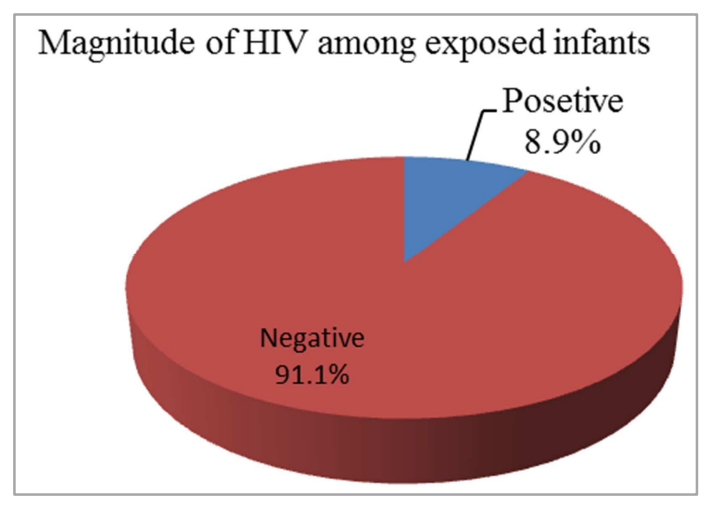

Figure 1. Magnitude of HIV among exposed infants in Sidama zone SNNPR Ethiopia, from 2014 - 2018 Gc.

\subsection{Characteristics of Study Participants}

The participants of this study were included from the 14 governmental hospitals found in Sidama zone SNNPR regional state in Ethiopia. A total of $203 \mathrm{HIV}$ exposed infant who had DNA /PCR test results and maternal Medical follow up card, were included in this study. More than half of the mothers $106(52.2 \%)$ were 25 to 34 years old. One hundred and eighteen $(58.1 \%)$ mothers resided in urban areas and the remaining $85(41.9 \%)$ lived in rural areas. The slightly more than half of HIV infected mothers, 107 (52.7\%) were primary school and $122(60.1 \%)$ mothers were House wife.

All mothers of HIV exposed infants were enrolled in obstetric care and support service during the last pregnancy. Among this 164 (80.8\%) women had attended ANC during Pregnancy, and slightly more than half 108 (53.2\%) had not completed TT5 immunization. Related to labour and delivery care service, $164(80.8 \%)$ of them were gave birth at health facility, majority of women $198(97.5 \%)$ had gave birth through spontaneous vaginal delivery (SVD), and 161 (79.3\%) had gave birth at term for last pregnancy. 
All HIV exposed infants who had DNA/PCR test results were enrolled in PMTCT of HIV care and support service during the follow -up period included in the study. From the study participants majority $162(79.8 \%)$ of HIV-exposed infants were enrolled into HIV care within six weeks of life. And also, $150(73.9 \%)$ of the infants were given ARV prophylaxis to prevent MTCT of HIV at birth. Most of HIVexposed infants received co-trimoxazole preventive therapy (CPT), and $162(79.8 \%)$ of infants started CPT at six weeks of being born. Regarding to infant feeding practice, 163 $(80.3 \%)$ infants were exclusive breastfed before six months. Regarding to EPI (expanded programs of immunization) all HIV exposed infant were immunized. The majority of this 149 (73.4\%) Infants were received Vitamin A. In this study all of HIV exposed infants mothers were enrolled in HIV care and support service during the last pregnancy. Out of these, $203(100 \%)$ of them were taking highly active treatment, $146(71.9 \%)$ of were known HIV status before PMTCT program Entry, $117(57.6 \%)$ baseline CD4 count were 350 and above, 134 (66.0\%) WHO clinical stages were Stage 3 and four during last pregnancy (Table 1).

\subsection{Factors Associated with MTCT of HIV Among Exposed Infants}

HIV disclosure status of mother, HIV Clinical stage of mother, Baseline CD4 count of mother, ART adherence, mother attend ANC during Pregnancy, Place of delivery, infant's age at diagnosis, infants received Vitamin A, infants received NVP at birth, infant feeding practice before six months have shown a significant association with HIV sero status of baby in the bivariate analysis at $\mathrm{P}$-value $<0.25$. After adjusting for common confounders, in the multivariate analysis, baseline CD4+ count of mother were less than 350 , maternal WHO clinical stage I and II, Poor ART adherence and infants who didn't receive Vitamin A had significant association to HIV sero status of infant at P-value of $<0.05$. Mothers whose baseline CD4 count less than 350 were 6 times $(\mathrm{AOR}=5.629 ; 95 \% \mathrm{CI}: 1.454,21.79)$ more likely to transmit HIV when compared with those with CD4 count greater than 350. Regarding to maternal WHO clinical staging, mothers who had WHO clinical stage I and II were $80 \%(\mathrm{AOR}=0.201 ; 95 \% \mathrm{CI}: 0.054,0.745)$ less likely transmit HIV compared with those who had stage III and IV. According to ART adherence, mothers Poor adherence to ART were 4 times $(\mathrm{AOR}=4.302 ; 95 \% \mathrm{CI}: 1.100,16.823)$ more likely to HIV compared with those who had good ART adherence. Infants not receiving Vitamin A at nine months were seven times (AOR=7.184; 95\% CI: 1.795, 28.759) more likely to have MTCT of HIV compared with Infants received Vitamin A at nine month of life. (Table 2)

Table 1. Socio demographic and clinical characteristics of HIV exposed infants and mothers in Sidama zone, SNNPR state, southern Ethiopia 2014-2018.

\begin{tabular}{|c|c|c|c|}
\hline Variables & & Frequency & Percentage \\
\hline \multirow{3}{*}{ Age of Mother } & $15-24 \mathrm{yr}$ & 46 & $22.7 \%$ \\
\hline & $25-34$ & 106 & $52.2 \%$ \\
\hline & $35-44$ & 51 & $25.1 \%$ \\
\hline \multirow{2}{*}{ Residence of Mother } & Urban & 118 & $58.1 \%$ \\
\hline & Rural & 85 & $41.9 \%$ \\
\hline \multirow{4}{*}{ Educational status of the Mother } & Illiterate & 54 & $26.6 \%$ \\
\hline & Primary & 107 & $52.7 \%$ \\
\hline & Secondary & 37 & $18.2 \%$ \\
\hline & College and above & 5 & $2.5 \%$ \\
\hline \multirow{4}{*}{ Occupation of Mother } & Farmer & 12 & $5.9 \%$ \\
\hline & Merchant & 59 & $29.1 \%$ \\
\hline & Government worker & 10 & $4.9 \%$ \\
\hline & House wife & 122 & $60.1 \%$ \\
\hline \multirow{4}{*}{ Religion of mothers } & Orthodox & 63 & $31.0 \%$ \\
\hline & Muslim & 84 & 41.4 \\
\hline & Protestant & 35 & 17.2 \\
\hline & Catholic & 21 & 10.3 \\
\hline \multirow{4}{*}{ Marital status of Mother } & Single & 16 & 7.9 \\
\hline & Married & 178 & 87.7 \\
\hline & Divorced & 5 & $2.5 \%$ \\
\hline & Windowed & 4 & $2 \%$ \\
\hline
\end{tabular}

\begin{tabular}{llc}
\hline Variables & Frequency & Percentage \\
\hline Obstetrics/ Reproductive Health history & & \\
Mother attended ANC during Pregnancy & 164 & $80.8 \%$ \\
Yes & 39 & $19.2 \%$ \\
No & 146 & $71.9 \%$ \\
Place of delivery & 57 & $28.1 \%$ \\
Health facility & 198 & $97.5 \%$ \\
Home delivery & 5 & $2.5 \%$ \\
Mode of delivery & & \\
SVD & & \\
Give birth at term pregnancy & \\
\hline
\end{tabular}




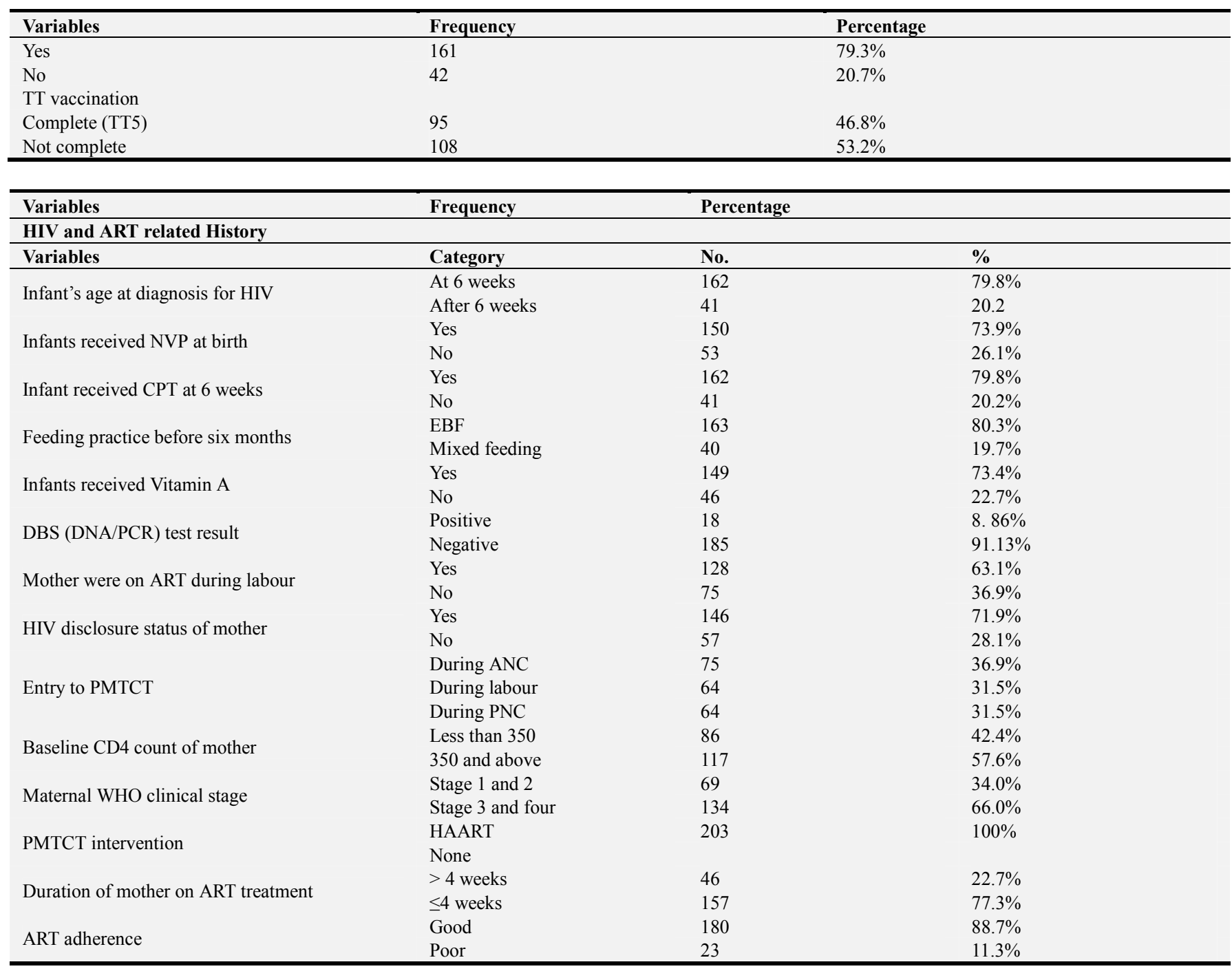

Table 2. Factors associated with HIV status among infants born to HIV positive mothers, in selected health facility of Sidama zones northern Ethiopia 2014 to January $2018 G c$

\begin{tabular}{|c|c|c|c|c|c|c|}
\hline \multirow[b]{2}{*}{ Variables } & \multicolumn{2}{|c|}{ DBS PCR/DNA Result } & \multirow{2}{*}{ COR $(95 \% \mathrm{CI})$} & \multirow[b]{2}{*}{ P-Value } & \multirow[b]{2}{*}{$\operatorname{AOR}(95 \% \mathrm{CI})$} & \multirow[b]{2}{*}{ P-Value } \\
\hline & Negative & Positive & & & & \\
\hline \multicolumn{7}{|c|}{ HIV disclosure status of mother } \\
\hline Yes (Ref) & $137(93.8 \%)$ & $9(6.2 \%)$ & & & & \\
\hline No & $48(84.2 \%)$ & $9(15.8 \%)$ & $2.85(1.07,7.61)$ & 0.036 & & \\
\hline \multicolumn{7}{|c|}{ HIV Clinical stage of mother } \\
\hline WHO stage I and II (Ref) & $59(85.5 \%)$ & $10(14.5 \%)$ & $0.37(.141,0.998)$ & 0.050 & $0.2(0.054,0.75)$ & $0.016^{*}$ \\
\hline WHO stage III and IV & $126(94.0 \%)$ & $8(6.0 \%)$ & & & & \\
\hline \multicolumn{7}{|c|}{ Baseline CD4 count of mother } \\
\hline Less than 350 & $74(86.0 \%)$ & $12(14.0 \%)$ & $3.0(1.07,8.346)$ & 0.035 & $5.63(1.45,21.79)$ & $0.012 *$ \\
\hline 350 and above (Ref) & $111(94.9 \%)$ & $6(5.1 \%)$ & & & & \\
\hline \multicolumn{7}{|l|}{ ART adherence } \\
\hline Good (Ref) & $168(93.3 \%)$ & $12(6.7 \%)$ & & & & \\
\hline Poor & $17(73.9 \%)$ & $6(26.1 \%)$ & $4.94(1.65,14.84)$ & 0.004 & $4.3(1.10,16.82)$ & $0.036^{*}$ \\
\hline \multicolumn{7}{|c|}{ Mother attend ANC during Pregnancy } \\
\hline Yes (Ref) & $153(93.3 \%)$ & $11(6.7 \%)$ & & & & \\
\hline No & $32(82.1 \%)$ & $7(17.9 \%)$ & $3.043(1.1,8.45)$ & 0.033 & & \\
\hline \multicolumn{7}{|l|}{ Place of delivery } \\
\hline Health facility (Ref) & $153(93.3 \%)$ & $11(6.7 \%)$ & & & & \\
\hline \multicolumn{6}{|l|}{ Infant's age at diagnosis } & \\
\hline At 6 weeks & $43(81.1 \%)$ & $10(18.9 \%)$ & $4.128(1.5,11.1)$ & 0.005 & & \\
\hline After 6 weeks (Ref) & $142(94.7 \%)$ & $8(5.3 \%)$ & & & & \\
\hline \multicolumn{7}{|l|}{ Infants received Vitamin A } \\
\hline Yes (Ref) & $140(94.0 \%)$ & $9(6.0 \%)$ & & & & \\
\hline
\end{tabular}




\begin{tabular}{|c|c|c|c|c|c|c|}
\hline \multirow{2}{*}{ Variables } & \multicolumn{2}{|c|}{ DBS PCR/DNA Result } & \multirow{2}{*}{ COR $(95 \%$ CI $)$} & \multirow{2}{*}{ P-Value } & \multirow{2}{*}{ AOR (95\% CI) } & \multirow{2}{*}{ P-Value } \\
\hline & Negative & Positive & & & & \\
\hline No & $38(82.6 \%)$ & $8(17.4 \%)$ & $3.28(1.18,9.06)$ & 0.022 & $7.18(1.79,28.8)$ & $0.005^{* *}$ \\
\hline \multicolumn{7}{|c|}{ Infants received NVP at birth } \\
\hline Yes (Ref) & $151(93.2 \%)$ & $11(6.8 \%)$ & & & & \\
\hline No & $34(82.9 \%)$ & $7(17.1 \%)$ & $2.83(1.02,7.8)$ & 0.045 & $3.43(0.96,12.26)$ & 0.058 \\
\hline \multicolumn{7}{|c|}{ Infant feeding practice before six months } \\
\hline EBF (Ref) & $152(93.3 \%)$ & $11(6.7 \%)$ & & & & \\
\hline Mixed feeding & $33(82.5 \%)$ & $7(17.5 \%)$ & $2.93(1.057,8.13)$ & 0.039 & $3.3(0.85,12.8)$ & 0.083 \\
\hline
\end{tabular}

\section{Discussion}

Prevention of Mother to child transmission of HIV needs early HIV diagnosis and timely preventive management. Polymerase chain reaction (PCR)-based HIV DNA and HIV RNA assays have become the most widely used, even in resource-limited settings, for both diagnostic and monitoring purposes [31]. This study was conducted to find out the magnitude of HIV status and associated factors among HIVExposed Infants' in Sidama zone, SNNPR state, southern Ethiopia.

In this study, the prevalence of HIV infections among exposed infants was 18 (8.9\%) 95\% CI: [4.4-12.8]. This reflects that the morbidity and mortality of the children is high due to this diseases condition. If appropriate preventive measures are not taking place, the risk of HIV among exposed infants might be ranked as tope five of infants' mortality. This finding was slightly higher than compared to the global target in 2015, which was 5\% among breastfeeding women [7] and also to be study conducted in East and West Gojjam Zones, Northwest Ethiopia shows that the prevalence of MTCT of HIV was $5.9 \%$ (3.9-7.9), [32] and the study conducted in Oromia Regional State $7.70 \%$ [23]. The possible explanation for this difference might be the difference in study design which most of them used cohort study design, sample size and study area. In addition to this, the gap might be due to the difference in the health care seeking behavior of infected mothers which in turn increases the recognition and prevention of MTCT. Despite the fact that the current health policy of Ethiopia focused on the implementation of option $\mathrm{B}+$ and free PMTCT service for infected mother and exposed infants; the prevalence of MTCT of HIV in our study is higher than the 2015 global target but lower than the systematic review and meta-analysis study conducted in Ethiopia $(9.93 \%$; $95 \%$ CI, $7.29,12.56$ ) [3]. This gap might be due to the differences in the study period, sample size, geographical difference of the study areas and health seeking behavior of the HIV infected mothers.

This study also revealed that factors associated with MTCT of HIV are: baseline CD4 count, WHO clinical stage of mother, ART adherence, and received Vitamin A. Mothers baseline $\mathrm{CD} 4+$ count less than 350 were six $(\mathrm{AOR}=5.629$; $95 \%$ CI: $1.454,21.79)$ times more likely to have an increased MTCT of HIV compared to those of Baseline CD4+ count greater than or equal to 350 . This reflects that having CD4+ count less than 350 increases the likelihood of MTCT of HIV. This finding was in line with the study conducted at
Zimbabwe which showed that maternal CD4 count less than 200 were more likely affect MTCT of HIV than counterpart (AOR=7.1; 95\% CI: 2.6-17) [19]. Our study also illustrated that mothers who had WHO clinical stages I and II were $80 \%$ (AOR $=0.201 ; 95 \%$ CI: $0.054,0.745)$ less likely to be MTCT of HIV compared with clinical stages III and IV. This reflects that mother having clinical stages I and II have minimum chance of MTCT of HIV. In the same line, a study conducted at northwest Ethiopia showed those in clinical stages I and II were four $(\mathrm{AOR}=4.4(\mathrm{CI}: 1.5,12)$ times more likely to prevent mother to child transmission of HIV compared with clinical stages III and IV [33]. Our study also demonstrated that poor ART adherence during last pregnancy four ( $\mathrm{AOR}=4.302 ; 95 \%$ CI: $1.100,16.823)$ times more likely affect MTCT of HIV compared to good ART adherence. This may indicate that there were significant numbers of women who might loss four and more doses within 60 days which could lead to poor ART adherence. This was incomparable with the study conducted in other area [23, 26]. The inconsistency might be due to the difference in the study area, study design, and health seeking behavior of the mothers. Numerous risk factors examined are strength of this study. Since the data was secondary, the information gathered was not complete in some infant's logbook and mother's medical registration card are limitations.

\section{Conclusion}

The prevalence of mother to child transmission of HIV was $18(8.9 \%)$. ART poor adherence, clinical stages I and II and low maternal CD4+ counts were factors associated with MTCT of HIV among exposed infants. Therefore, relevant stakeholders should make efforts to reduce the magnitude of MTCT of HIV through strengthening the prevention of mother-to-child transmission (PMTCT) of HIV, and health education for infected mother on the importance of ART adherence and undertaking further study are recommended.

\section{List of Abbreviation / Acronym}

AIDS: Acquired immune deficiency virus

ART: Ant retro viral therapy

CD4+: Cluster of differentiation four plus

HIV: Human immunodeficiency virus

IRV: Institutional review board

JU: Jimma University

MTCT: Mother to child transmission o

PMTCT: Prevention mother to child transmission 
PCR: Polymerase chain reaction

SVD: Spontaneous vaginal delivery

UNSAID: Unitized state aid for international development WHO: World health organization

SNNPR: South nation nationality and people region

\section{Ethics Approval and Consent to Participate}

Ethical clearance for the study was obtained from the Jimma university, institutional review board (IRB) of Jimma University Ethical Review Board (Nu85/8050/2018Gc). The permission letter was obtained from SNNPR Health bureau and Sidama zone health bureau administrative office and delivered to the respective study institutions. Data were processed using unique identifiers for purpose of privacy and confidentiality.

\section{Authors' Contribution}

YY, BT, JJ and SA, contributed to the planning and development of the study design. YY was responsible for the data collection. YY, BT, JJ and SA were involved in the data analysis and interpretation. YY wrote the first draft of the manuscript with significant contributions from all authors. All the co-authors edited and approved the final version.

\section{References}

[1] WHO. UNAIDS report on the global AIDS epidemic 2013.

[2] EPHIA. Ethiopia population-based hiv impact assessment ephia 2017-2018.

[3] Kassa GM. Mother-to-child transmission of HIV infection and its associated factors in Ethiopia: a systematic review and meta-analysis. BMC Infect Dis. 2018; 18 (1): 216.

[4] WHO. Elimination of mother-to-child transmission of hiv and syphilis. 2017.

[5] WHO. HIV prevention programmes overview. 2017.

[6] WHO. Programmatic update use of antiretroviral drugs for treating pregnant women and preventing hiv infection in infants. 2013

[7] WHO. Prevention of mother-to-child transmission (PMTCT) of HIV >. 2018 (08): 20.

[8] Etoori D, Kerschberger B, Staderini N, Ndlangamandla M, Nhlabatsi B, Jobanputra K, et al. Challenges and successes in the implementation of option $\mathrm{B}+$ to prevent mother-to-child transmission of HIV in southern Swaziland. BMC Public Health. 2018; 18 (1): 374.

[9] WHO. Preventing mother-to-child transmission of HIV to reach the UNGASS and Millennium Development Goals. 2010 .

[10] WHO. Prevention of mother-to-child transmission (PMTCT) of HIV. 2019.

[11] WHO. Eliminating Mother-to-Child Transmission of HIV by
2030: 5 Strategies to Ensure Continued Progress.

[12] Deressa W, Seme A, Asefa A, Teshome G, Enqusellassie F. Utilization of PMTCT services and associated factors among pregnant women attending antenatal clinics in Addis Ababa, Ethiopia. BMC Pregnancy Childbirth. 2014; 14 (14): 328.

[13] FMOH. National aids and sti's control programme federal ministry of health 2016.

[14] EDHS. Demographic and Health Survey HIV Prevalence Report. 2016.

[15] FDRE. Federal Democratic Republic of Ethiopia Ministry of Health for comprehensive hiv prevention, care and treatment national guidelines for comprehensive hiv prevention, care and treatment 2014.

[16] Prevention of mother-to-child HIV transmission cascade in China: a systematic review and meta-analysis. 2014.

[17] Esther A, Nlend N, Motaze ACN. HIV-1 transmission and survival according to feeding options in infants born to HIVinfected women in Yaoundé, Cameroon. 2018.

[18] Mwau M, Bwana P, Kithinji L, Ogollah F, Ochieng S, Akinyi C, et al. Mother-to-child transmission of HIV in Kenya: A cross-sectional analysis of the national database over nine years. PLoS One. 2017; 12 (8): e0183860.

[19] Ngwende S, Gombe NT, Midzi S. Factors associated with HIV infection among children born to mothers on the prevention of mother to child transmission programme at Chitungwiza Hospital, Zimbabwe. 2013.

[20] Endalamaw, Aklilu, Demsie. A systematic review and metaanalysis of vertical transmission route of HIV in Ethiopia. 2018.

[21] Rate of HIV transmission and associated factors among HIVexposed infants in selected health facilities of East and West Gojjam Zones, Northwest Ethiopia; retrospective cohort study. 2017.

[22] Wudineh F, Damtew B. Mother-to-Child Transmission of HIV Infection and Its Determinants among Exposed Infants on Care and Follow-Up in Dire Dawa City, Eastern Ethiopia. AIDS Res Treat. 2016; 3262746.

[23] Obsa S, Dabsu R, Ejeta E. Rate of mother to child transmission of HIV and factors associated among HIV exposed infants in Oromia Regional State, Ethiopia: Retrospective study. Egyptian Pediatric Association Gazette. 2018; 66 (3): 61-5.

[24] Derebe G, Biadgilign S, Trivelli M. Determinant and outcome of early diagnosis of HIV infection among HIV-exposed infants in southwest Ethiopia. 2014.

[25] Wondafrash B, DH. Dried blood spot test for hiv exposed infants and children and their anti-retro viral treatment status in selected hospitals in ethiopia. 2016.

[26] Gebremedhin A, Gebremariam S, Haile F. Predictors of mortality among HIV infected children on anti-retroviral therapy in Mekelle Hospital, Northern Ethiopia: a retrospective cohort study. 2013.

[27] Beyene GA, Dadi LS, Solomon a. Determinants of HIV infection among children born to mothers on prevention of mother to child transmission program of HIV in Addis Ababa, Ethiopia: a case control study. 2018. 
[28] Mother-to-child transmission of HIV infection and its associated factors in Ethiopia: a systematic review and metaanalysis. 2018.

[29] Berhan Z, Abebe F, Gedefaw M. Risk of HIV and associated factors among infants born to HIV positive women in Amhara region, Ethiopia: a facility based retrospective study. 2014.

[30] SNNPR. Facility Type - SNNP Regional Health Bureau 2016.

[31] WHO. Who recommendations on the diagnosis of hiv infection in infants and children. 2010.
[32] Moges NA, Kassa GM, Boneya DJ. Rate of HIV transmission and associated factors among HIV-exposed infants in selected health facilities of East and West Gojjam Zones, Northwest Ethiopia; retrospective cohort study. BMC Infect Dis. 2017; 17 (1): 475.

[33] Ketemaw A. Risk of HIV and associated factors among infants born to HIV-positive women in northwest Ethiopia. 